\title{
Efficient Evaluation of Fourier-Based SAR Focusing Kernels
}

\author{
Pau Prats-Iraola, Senior Member, IEEE, Marc Rodriguez-Cassola, Francesco De Zan, \\ Paco López-Dekker, Member, IEEE, Rolf Scheiber, Andreas Reigber, Senior Member, IEEE
}

\begin{abstract}
This paper addresses the efficient evaluation of Fourier-based kernels for synthetic aperture radar (SAR) image formation. The goal is to evaluate the quality of the focused impulse response function and the residual phase errors of the kernel without having to implement the processor itself nor perform a costly point-target simulation followed by the processing. The proposed methodology is convenient for situations where the assumption of a hyperbolic range history does not hold anymore, and hence a compact analytic expression of the point target spectrum is not available. Examples where the hyperbolic range history does not apply include very highresolution spaceborne SAR imaging or bistatic SAR imaging. The approach first computes numerically the two-dimensional (2D) spectrum of a point target and then uses the transfer function of the focusing kernel to match it. The spectral support is then computed to adapt the spectrum to the output imaging geometry, so that the impulse response function (IRF) is finally obtained. The proposed approach is valid under the assumption of a large time-bandwidth product, as is usually the case for current airand spaceborne SAR sensors. The methodology is validated by comparing the matched IRFs with the ones obtained using pointtarget simulations.
\end{abstract}

Index Terms-Synthetic aperture radar (SAR), Spotlight SAR, SAR processing, SAR spectrum, SAR Simulation.

\section{INTRODUCTION}

Future spaceborne synthetic aperture radar (SAR) systems are being developed in order to deliver a better performance, e.g., in terms of spatial resolution and coverage. The use of satellite constellations is also an intense research topic, where bistatic imaging can help to further improve the aforementioned aspects. In terms of the SAR raw data focusing, high resolution and bistatic imaging are demanding, especially when the range history can no longer be expressed analytically in a compact form. In such cases, the evaluation of the focusing algorithm is usually performed via time-domain simulation of point targets followed by the processing of the raw data using the implemented focusing kernel. This is expensive in computational terms, which also limits the number of scenarios that can be simulated. However, during the assessment of a spaceborne SAR mission, it is desirable to efficiently validate the performance of the focusing kernel for all possible scenarios, e.g., different incidence angles or scene sizes.

This paper presents a methodology to quantify the performance of a focusing kernel without having to implement the processor itself nor perform a costly point-target simulation.

Manuscript received xxxx; revised xxxx

P. Prats-Iraola, and the co-authors are with the Microwaves and Radar Institute, German Aerospace Center (DLR), Oberpfaffenhofen D-82234, Germany (e-mail: Pau.Prats@dlr.de).
The methodology is based on the computation of the transfer function of the focusing kernel, and hence convenient for Fourier-based processing algorithms. This transfer function is compared to the frequency responses of the point targets, which are computed numerically using their range histories. The difference can be directly evaluated to assess the performance of the focusing kernel. The methodology is valid as long as a large time-bandwidth product applies, which is the usual case in current air- and spaceborne SAR systems. The same rationale was presented in [1] by deriving the transfer function of several kernels analytically. Such analytic evaluation assumes a single-platform linear-track geometry, which is not a valid model for many future SAR missions, e.g., bistatic or very high resolution. For this reason the numerical evaluation of the kernel is introduced in this letter. The proposed approach evaluates also inherently the capability of the kernel to accommodate particular aspects of the geometry, like the azimuth variance or the topography dependence.

For the presented methodology it is not relevant how some steps within the processing are performed. For example, the fact that the chirp scaling algorithm equalizes the range curvatures using the chirp scaling principle [2], or that an interpolation is implemented via chirp- $Z$ transform [3], will not affect the transfer function itself, since it is assumed that these steps are performed with arbitrary accuracy. Similarly, pre- and post-processing steps in order to handle spectral or time aliasing, as it happens in the spotlight [3]-[5], ScanSAR [2] or TOPS [5] modes, are not meant to be evaluated with the proposed approach, as again there is no reason not to perform these steps accurately. It is important to remark that the proposed methodology is not intended to replace the conventional evaluation of a processor via time-domain simulation of point targets, but rather to complement it.

Section II expounds the main aspects of the proposed approach, namely, the computation of the point target's spectrum, the evaluation of the transfer function of the focusing kernel, and the spectral support. Section III summarizes the methodology, and Section IV validates it using time-domain point-target simulations.

\section{Evaluation OF THE FocUsing KeRnEL}

\section{A. Computation of the Point Target's Spectrum}

In order to compute analytically the two-dimensional (2D) spectrum of a range-compressed point target, the first step is a Fourier transform (FT) in the range dimension. This FT, 
neglecting the amplitude terms, is given by [6]-[8]

$$
H\left(f_{\mathrm{r}}, t_{\mathrm{a}}\right)=\exp \left[-\mathrm{j} \cdot \frac{2 \pi}{\mathrm{c}} \cdot\left(f_{0}+f_{\mathrm{r}}\right) \cdot R\left(t_{\mathrm{a}}\right)\right],
$$

where $f_{\mathrm{r}}$ is the range frequency, $t_{\mathrm{a}}$ is the azimuth time, $\mathrm{c}$ is the speed of light, $f_{0}$ is the central frequency, and $R\left(t_{\mathrm{a}}\right)$ is the two-way range history. The azimuth FT can then be computed asymptotically using the principle of stationary phase [9], which requires the computation of the stationary time, $t_{\mathrm{a}}^{*}$, being the one satisfying [6]

$$
\left.\frac{\partial R\left(t_{\mathrm{a}}\right)}{\partial t_{\mathrm{a}}}\right|_{t_{\mathrm{a}}=t_{\mathrm{a}}^{*}}=-\frac{\mathrm{c} \cdot f_{\mathrm{a}}}{f_{0}+f_{\mathrm{r}}},
$$

where $f_{\mathrm{a}}$ is the azimuth frequency. After evaluating (2) for each point of the 2D spectrum, the phase of the FT of (1) can be approximated by

$$
\varphi_{\mathrm{T}}\left(f_{\mathrm{r}}, f_{\mathrm{a}}\right) \approx-\frac{2 \pi}{\mathrm{c}} \cdot\left(f_{0}+f_{\mathrm{r}}\right) \cdot R\left(t_{\mathrm{a}}^{*}\right)-2 \pi \cdot f_{\mathrm{a}} \cdot t_{\mathrm{a}}^{*},
$$

where the range history needs to be evaluated at each $t_{\mathrm{a}}^{*}$.

In some special cases $t_{\mathrm{a}}^{*}$ can be solved analytically, e.g., when the range history is hyperbolic, which results in the wellknown compact expression of the 2D phase spectrum [1], [6], [10]. However, for the cases under consideration in this letter, (2) needs to be solved numerically using, e.g., the NewtonRapshon method, or series reversion by first expressing $R\left(t_{\mathrm{a}}\right)$ as a power series of $t_{\mathrm{a}}$ [7]. Indeed, series reversion has been used in the literature to compute the focusing kernel for bistatic imaging [7] and high-resolution SAR imaging [8].

Under the assumption that $\varphi_{\mathrm{T}}\left(f_{\mathrm{r}}, f_{\mathrm{a}}\right)$ is an accurate representation of the target's $2 \mathrm{D}$ spectrum phase, any processing kernel can be evaluated by comparing its transfer function to that of the point target, as shown in the following section.

\section{B. Computation of the Transfer Function of the Kernel}

The transfer function can be obtained either from the expressions of the processor found in the literature, or by computing it in case of numerical kernels. Note that Fourierbased SAR image formation is based on phasor multiplications and interpolations, so that a generic transfer function of a Fourier-based focusing kernel can be expressed as follows

$$
\begin{aligned}
H_{\mathrm{K}}\left(f_{\mathrm{r}}, f_{\mathrm{a}}\right)= & W\left(f_{\mathrm{r}}, f_{\mathrm{a}}\right) \cdot \exp \left[\mathrm{j} \cdot \varphi_{\mathrm{F}}\left(f_{\mathrm{r}}, f_{\mathrm{a}}\right)\right] \\
& \cdot \exp \left[\mathrm{j} \cdot 2 \pi \cdot \Delta t_{\mathrm{r}}\left(f_{\mathrm{a}}\right) \cdot f_{\mathrm{r}}\right] \\
& \cdot \exp \left[\mathrm{j} \cdot \varphi_{\mathrm{ac}}\left(f_{\mathrm{a}}\right)\right],
\end{aligned}
$$

where $W(\cdot)$ represents the weighting function for sidelobe suppression, and $\varphi_{\mathrm{F}}(\cdot)$ is the matched filter at the given range including the range cell migration correction (RCMC) and higher order terms. The phase ramp of the second exponential term with $\Delta t_{\mathrm{r}}$ represents an interpolation in the range-time, azimuth-frequency domain to correct for any residual term of the range cell migration (RCM). The last exponential term with $\varphi_{\text {ac }}$ represents a residual azimuth compression. The last two might be needed depending on the geometry and the selected processing approach, as for example occurs in the spaceborne case due to the dependence on the effective velocixty with range [6], [8], [11]. The complete phase of the transfer function is defined as $\varphi_{\mathrm{K}}=\arg \left\{H_{\mathrm{K}}\right\}$. Note that in (4) the dependence on the target's position, $\left(r_{0}, t_{0}\right)$, has been omitted for simplicity, being $r_{0}$ the closest approach distance and $t_{0}$ the zero-Doppler time at closest approach.

Operations not performed in the 2D frequency domain need to be considered differently. It has already been shown how to handle interpolations in the fast-time domain. Azimuthindependent phase corrections are also trivial to map, while azimuth-variant phase corrections need to be specially treated. Consider a correction in the slow-time domain given by $\alpha\left(t_{\mathrm{a}} ; f_{\mathrm{r}}\right)$, which might depend on the range frequency. If the correction is very slow variant, one can assume that the stationary phase point computed with (2) will not change significantly. In this case, the correction in the 2D frequency domain is directly given by $\alpha\left(t_{\mathrm{a}}^{*} ; f_{\mathrm{r}}\right)$. However, a more precise approach is to compute a new stationary phase point, $t_{\mathrm{a}}^{\star}$, so that (2) is substituted by

$$
\left.\frac{\partial}{\partial t_{\mathrm{a}}}\left\{-\frac{2 \pi}{\mathrm{c}} \cdot\left(f_{0}+f_{\mathrm{r}}\right) \cdot R\left(t_{\mathrm{a}}\right)+\alpha\left(t_{\mathrm{a}} ; f_{\mathrm{r}}\right)\right\}\right|_{t_{\mathrm{a}}=t_{\mathrm{a}}^{\star}}=2 \pi \cdot f_{\mathrm{a}},
$$

and the correction in the 2D frequency domain is then given by $\alpha\left(t_{\mathrm{a}}^{\star} ; f_{\mathrm{r}}\right)$ and the target's spectrum in (3) shall be also evaluated at $t_{\mathrm{a}}^{\star}$.

Finally, the phase error, $\varphi_{\text {error }}\left(f_{\mathrm{r}}, f_{\mathrm{a}}\right)$, is obtained by subtracting the kernel's phase, (4), to the computed target's phase, (3). In the presence of an azimuth-variant phase correction the stationary phase point given by (5) shall be used instead of (2). Two transfer functions of state-of-the-art kernels are shown later in Section II-E.

\section{Shaping the Spectral Support}

The impulse response function (IRF) is given by the phase error and the selected weighting. However, the transfer function in (4) does not consider the range-variant property of the geometry. Indeed, such a matched filter will focus a target at the given range, but other targets will be more defocused, the larger their distance to the reference one. Accurate SAR processors accommodate the range-variant geometry, a step that warps the spectrum and, consequently, defines the spectral support of the focused image. Taking as example the rangeDoppler algorithm, its azimuth compression filter changes with range, which introduces a Doppler-dependent phase ramp in the fast time, and hence a Doppler-dependent shift in the range-frequency domain [12], which curves the spectrum of the target. It is even clearer to visualize this effect through the $\omega$-k algorithm, since the Stolt interpolation performs this warp directly. Note though, that the main contribution in the shift of the range spectrum comes from the range-variant azimuth compression filter, resulting in a range spectral shift given by

$$
\Delta f_{\mathrm{r}}\left(r, f_{\mathrm{a}}\right)=\frac{\mathrm{c}}{2 \pi} \frac{\partial \varphi_{\mathrm{ac}}\left(r, f_{\mathrm{a}}\right)}{\partial r},
$$

where $r$ is the range vector. In the hyperbolic monostatic case, (6) is given by [12]

$$
\begin{aligned}
\Delta f_{\mathrm{r}, \mathrm{m}}\left(f_{\mathrm{a}}\right) & =-f_{0} \cdot\left(1-\sqrt{1-\left(\frac{\lambda \cdot f_{\mathrm{a}}}{2 \cdot v_{\mathrm{e}}}\right)^{2}}\right) \\
& =-f_{0} \cdot\left[1-\gamma\left(f_{\mathrm{a}}, v_{\mathrm{e}}\right)\right],
\end{aligned}
$$




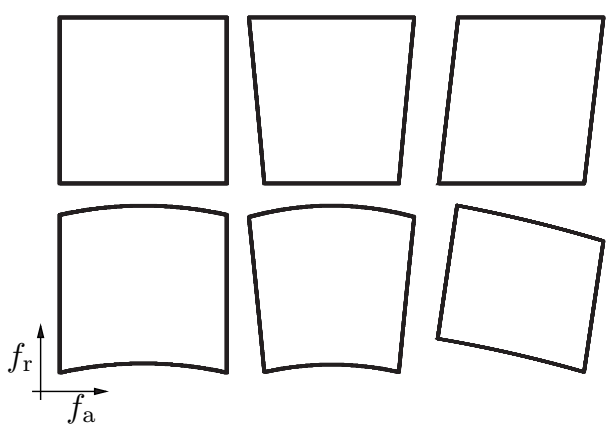

Fig. 1. Spectral support at (top row) raw data level and (bottom row) image level for (left column) non-squinted stripmap, (middle column) non-squinted spotlight and (right column) squinted stripmap.

where $v_{\mathrm{e}}$ is the effective velocity and $\gamma\left(f_{\mathrm{a}}, v_{\mathrm{e}}\right)$ is the azimuth modulation term. A low-order interpolator can be used to interpolate the phase values and the envelope separately.

An aspect affecting only the spectrum envelope is the wavelength dependence and the truncation of the raw data in time-domain. Considering an equivalent antenna pattern that falls to zero at half of the beamwidth, the raw data spectrum of a stripmap acquisition is square, but that of a spotlight acquisition is a trapezoid. This can be shown by recalling the well-known relation of the antenna beamwidth with the wavelength and the azimuth antenna length, $L_{\mathrm{a}}$, given by $\theta_{\mathrm{a}} \propto \lambda / L_{\mathrm{a}}$, where a linear dependence on the wavelength is assumed to be valid. This relation implies that, since the antenna length is fixed, it "sees" the target first at lower frequencies than at higher ones. Precisely due to this wavelength dependence, it can be stated that the nominal azimuth resolution of a stripmap SAR is half of the antenna length. This fact implies automatically that the raw data spectrum must be square for a stripmap acquisition. However, as soon as the data are truncated in the slow-time domain, as it happens for example in a spotlight acquisition, the azimuth instantaneous bandwidth is wavelength dependent, resulting in the trapezoidal form of the spectrum. Indeed, a time-domain simulation of a point target usually neglects the wavelength dependence of the antenna beam, yielding the trapezoidal form that can often be seen in the literature, which is only strictly correct for the spotlight mode. The first two columns of Fig. 1 show the shape of the spectrum at raw- and image-data levels for the stripmap and spotlight modes, where in the latter case the trapezoidal form due to time truncation can be appreciated.

A final aspect that only affects the envelope of the spectrum results from the relation between Doppler frequency and time given by

$$
f_{\mathrm{DC}}=-\left.\frac{1}{\lambda} \cdot \frac{\partial R\left(t_{\mathrm{a}}\right)}{\partial t_{\mathrm{a}}}\right|_{t_{\mathrm{a}}=t_{\mathrm{c}}},
$$

where $t_{\mathrm{c}}$ is the beam-center time. In the monostatic case, eq. (8) takes the well-known expression of $f_{\mathrm{DC}}=2 \cdot v_{\mathrm{p}} \cdot \sin \beta / \lambda$, where $\beta$ is the squint angle and $v_{\mathrm{p}}$ is the platform velocity. Due to the wavelength dependence, the raw data spectrum is skewed [13]. This is shown in the third column of Fig. 1, where the spectral support of a squinted stripmap acquisition is sketched before and after image formation.

\section{Evaluation of the Results}

In general, the phase error $\varphi_{\text {error }}$ has a low-pass character (i.e., $\Delta f \cdot\left|\partial \varphi_{\text {error }} / \partial f_{\mathrm{a}}\right|<\pi$, being $\Delta f$ the frequency bin in Hertz), so that the computation of $\varphi_{\mathrm{T}}$ and $\varphi_{\mathrm{K}}$ can be done using few samples, further allowing one to put the phase error into an exponential term and perform an inverse FT in order to obtain the IRF for the point being evaluated, i.e.,

$$
h\left(t_{\mathrm{r}}, t_{\mathrm{a}}\right)=\mathrm{FT}_{2 \mathrm{D}}^{-1}\left\{\Gamma\left\{W\left(f_{\mathrm{r}}, f_{\mathrm{a}}\right) \cdot \exp \left[\mathrm{j} \cdot \varphi_{\text {error }}\left(f_{\mathrm{r}}, f_{\mathrm{a}}\right)\right]\right\}\right\},
$$

where the $\Gamma\{\cdot\}$ operator represents the shaping of the spectrum expounded in the previous section. The usual parameters of interest can now be measured, namely, resolution, peakto-sidelobe ratio, integrated-sidelobe ratio, pixel-shift error, etc. The phase value at the maximum of the IRF gives the (interferometric) phase error, which should be ideally $0^{\circ}$.

\section{E. Kernel Examples}

In order to give some more insight into the methodology, an example of the transfer function is shown next for the monostatic range-Doppler (RD) and the $\omega$-k algorithms. Starting with $\mathrm{RD}$, the transfer function is given by [6]

$$
\begin{aligned}
\varphi_{\mathrm{RD}}= & -\frac{4 \pi}{\lambda} \cdot r_{\mathrm{ref}} \cdot \sqrt{\left(1+\frac{f_{\mathrm{r}}}{f_{0}}\right)^{2}-\left(\frac{\lambda \cdot f_{\mathrm{a}}}{2 \cdot v_{\mathrm{e}, \mathrm{ref}}}\right)^{2}} \\
& +2 \pi \cdot \Delta t_{\mathrm{r}, \mathrm{RD}}\left(f_{\mathrm{a}}\right) \cdot f_{\mathrm{r}} \\
& -\frac{4 \pi}{\lambda} \cdot\left[r_{0} \cdot \gamma\left(f_{\mathrm{a}}, v_{\mathrm{e}}\right)-r_{\mathrm{ref}} \cdot \gamma\left(f_{\mathrm{a}}, v_{\mathrm{e}, \mathrm{ref}}\right)\right],(
\end{aligned}
$$

where $r_{\text {ref }}$ is the closest approach distance for the reference target, $v_{\mathrm{e}, \text { ref }}$ is the effective velocity of the reference range, and the residual RCM is given by

$$
\Delta t_{\mathrm{r}, \mathrm{RD}}\left(f_{\mathrm{a}}\right)=-\frac{2}{\mathrm{c}} \cdot\left(\frac{r_{0}}{\gamma\left(f_{\mathrm{a}}, v_{\mathrm{e}}\right)}-\frac{r_{\mathrm{ref}}}{\gamma\left(f_{\mathrm{a}}, v_{\mathrm{e}, \mathrm{ref}}\right)}\right) .
$$

The first term in (10) is the matched filter performed in the $2 \mathrm{D}$ frequency domain at the beginning of the processing tuned at $r_{\text {ref }}$, the second term performs the residual range cell migration, while the last one performs the residual azimuth compression. After computing $\varphi_{\text {error }}$, the warp of the spectrum as given by (7) shall be introduced using a low-order interpolator.

For the $\omega$-k case, the transfer function is [6], [10]

$$
\begin{aligned}
\varphi_{\omega \mathrm{k}}= & -\frac{4 \pi}{\lambda} \cdot r_{0} \cdot \sqrt{\left(1+\frac{f_{\mathrm{r}}}{f_{0}}\right)^{2}-\left(\frac{\lambda \cdot f_{\mathrm{a}}}{2 \cdot v_{\mathrm{e}, \mathrm{ref}}}\right)^{2}} \\
& +2 \pi \cdot \Delta t_{\mathrm{r}, \omega \mathrm{k}}\left(f_{\mathrm{a}}\right) \cdot f_{\mathrm{r}} \\
& -\frac{4 \pi}{\lambda} \cdot r_{0} \cdot\left[\gamma\left(f_{\mathrm{a}}, v_{\mathrm{e}}\right)-\gamma\left(f_{\mathrm{a}}, v_{\mathrm{e}, \mathrm{ref}}\right)\right],
\end{aligned}
$$

where the residual RCM is given by

$$
\Delta t_{\mathrm{r}, \omega \mathrm{k}}\left(f_{\mathrm{a}}\right)=-\frac{2 \cdot r_{0}}{\mathrm{c}} \cdot\left(\frac{1}{\gamma\left(f_{\mathrm{a}}, v_{\mathrm{e}}\right)}-\frac{1}{\gamma\left(f_{\mathrm{a}}, v_{\mathrm{e}, \mathrm{ref}}\right)}\right) .
$$

The warp can be performed using the Stolt mapping with a low-order interpolator.

In the case of numerical kernels, be it monostatic [8] or bistatic [14], the transfer function can be evaluated numerically, but a similar structure as the examples before will 


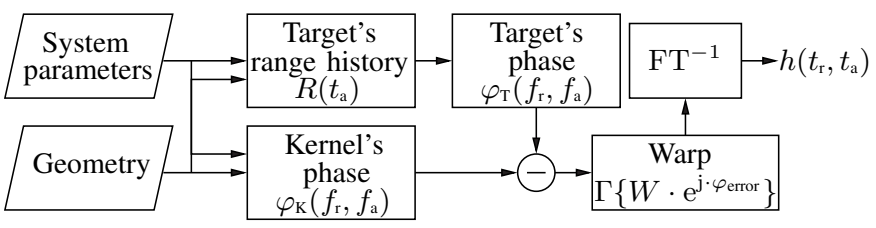

Fig. 2. Flow of the proposed methodology. The dependence of $R(\cdot)$, $\varphi_{\mathrm{K}}(\cdot), \varphi_{\mathrm{T}}(\cdot)$ and $h(\cdot)$ with the target position $\left(r_{0}, t_{0}\right)$ has been omitted for simplicity, as well as the dependence of $W(\cdot)$ and $\varphi_{\text {error }}(\cdot)$ with $\left(f_{\mathrm{r}}, f_{\mathrm{a}} ; r_{0}, t_{0}\right)$.

remain, namely, a matched filter, a residual RCMC, and a residual azimuth compression, where the last two are optional depending on the algorithm.

\section{Proposed Methodology}

Fig. 2 shows the flow of the proposed methodology to evaluate a given Fourier-based SAR focusing kernel. A preliminary step is the definition of the geometry, e.g., sensor(s) trajectory(ies), azimuth and range scene extensions, number of targets and their positions within the imaged scene; and of the system parameters, e.g., central frequency, bandwidths, mode. The next step is the computation of the range history $R\left(t_{\mathrm{a}}\right)$ for each target, which is obtained using the sensor(s) trajectory(ies) and the target's position. Then, the target's phase in the $2 \mathrm{D}$ frequency domain, $\varphi_{\mathrm{T}}\left(f_{\mathrm{r}}, f_{\mathrm{a}}\right)$, is computed as described in Section II-A. In parallel, the kernel's transfer function phase for the given target, $\varphi_{\mathrm{K}}\left(f_{\mathrm{r}}, f_{\mathrm{a}}\right)$, is also obtained, a step that besides the system parameters needs also the range history or associated values, e.g., closest approach distances and effective velocities. For numerical kernels, the range history(ies) of the reference target(s) might be also needed. The efficiency of the proposed approach lies in the fact that both $\varphi_{\mathrm{T}}$ and $\varphi_{\mathrm{K}}$ are smooth functions, while $\varphi_{\text {error }}$ is assumed to be low pass, and therefore they all can be modeled accurately with few samples. Once $\varphi_{\text {error }}$ is computed, the shaping (warping) of the spectral support is performed as described in Section II-C. After combining the amplitude and the phase, an inverse FT yields the IRF, which can be evaluated as usual.

\section{EXPERIMENTAL VALIDATION}

This section demonstrates the methodology by comparing its output with time-domain point-target simulations followed by the true processing. Two different examples are shown: a monostatic spotlight geometry, and an airborne-spaceborne bistatic geometry (see Table I). The selected size of the target's spectra and the corresponding matched filters computed with the proposed methodology has been of just $64 \times 64$ samples, while the target's range history has been fitted with a sixth order polynomial before performing series reversion.

For the spotlight example, the range-Doppler and the extended $\omega-\mathrm{k}$ (EOK) [15] approaches are evaluated, where the latter has been adapted to the spaceborne scenario [11]. The matched filters are the ones shown in (10) and (12), respectively. Due to the very high resolution, conventional kernels assuming a hyperbolic range history do not achieve
TABLE I

SPOTLIGHT AND BISTATIC SIMULATION PARAMETERS

\begin{tabular}{lcc}
\hline Parameter & Spotlight & Bistatic \\
\hline Central frequency & $9.65 \mathrm{GHz}$ & $9.65 \mathrm{GHz}$ \\
Integration time & $\sim 8 \mathrm{sec}$ & $\sim 3 \mathrm{sec}$ \\
Total az. proc. bandwidth & $33 \mathrm{kHz}$ & $7 \mathrm{kHz}$ \\
Azimuth resolution & $19 \mathrm{~cm}$ & $23 \mathrm{~cm}$ \\
Chirp bandwidth & $1.2 \mathrm{GHz}$ & $150 \mathrm{MHz}$ \\
Ground range scene size & $5 \mathrm{~km}$ & $2.5 \mathrm{~km}$ \\
Azimuth scene size & $5 \mathrm{~km}$ & $500 \mathrm{~m}$ \\
Spacecraft incidence angle & $55^{\circ}$ & $51^{\circ}$ \\
Aircraft incidence angle & - & $45^{\circ}$ \\
Aircraft velocity & - & $90 \mathrm{~m} / \mathrm{s}$ \\
Aircraft altitude & - & $2180 \mathrm{~m}$ \\
\hline
\end{tabular}

a satisfactory focusing performance. For this reason, the orbit compensation suggested in [11] is used, which corrects the signal in terms of phase and envelope. Since this correction is performed in the azimuth-time, range-frequency domain, the stationary point is computed using (5) in order to obtain the orbit compensation correction and the target's phase history in the $2 \mathrm{D}$ frequency domain. The true processing was performed using subapertures and a spectral analysis (SPECAN) approach was used for the azimuth processing [4], [11]. Fig. 3 shows the obtained IRFs and phase errors of the target located at far range at the edge of the scene. The difference in the phase error in the 2D frequency domain between the proposed methodology and the true processing is practically zero, but for the Gibbs phenomenon at spectrum edges. The computation of the IRFs for the nine targets located in the scene took less than a minute with the proposed approach, while the true processing lasted more than four hours in a sixteen-core computer.

Concerning the bistatic example, its geometry corresponds to the experiment that took place on November 2007 between the TerraSAR-X satellite and DLR's F-SAR airborne system [16]. The selected kernel is a numerical range-Doppler algorithm, where the point of stationary phase has been computed using series reversion [7]. Despite being a monochromatic algorithm, it performs well due to the small swath and relative small bandwidth. However, the azimuth-variant characteristic is not accommodated. Fig. 4 shows the result for a target located at the same range as the reference target but $125 \mathrm{~m}$ away from it in the azimuth dimension. Again, the obtained result compared to the true processing is practically identical.

\section{CONCLUSION}

This paper has presented a methodology to efficiently evaluate the performance of Fourier-based SAR focusing kernels. Such methodology is convenient for imaging geometries where a close-form analytic expression of the signal in the two-dimensional frequency domain is not possible, as for example occurs in bistatic or very high resolution spaceborne geometries. Due to its high efficiency, it is straightforward to evaluate different mission scenarios, e.g., different latitudes and incidence angles, different spaceborne constellations, or to quantify other aspects like the influence of the topography or the azimuth variance. The outcome is a global performance of the focusing kernel, hence becoming a powerful tool for the final selection of the focusing kernel that best suits 

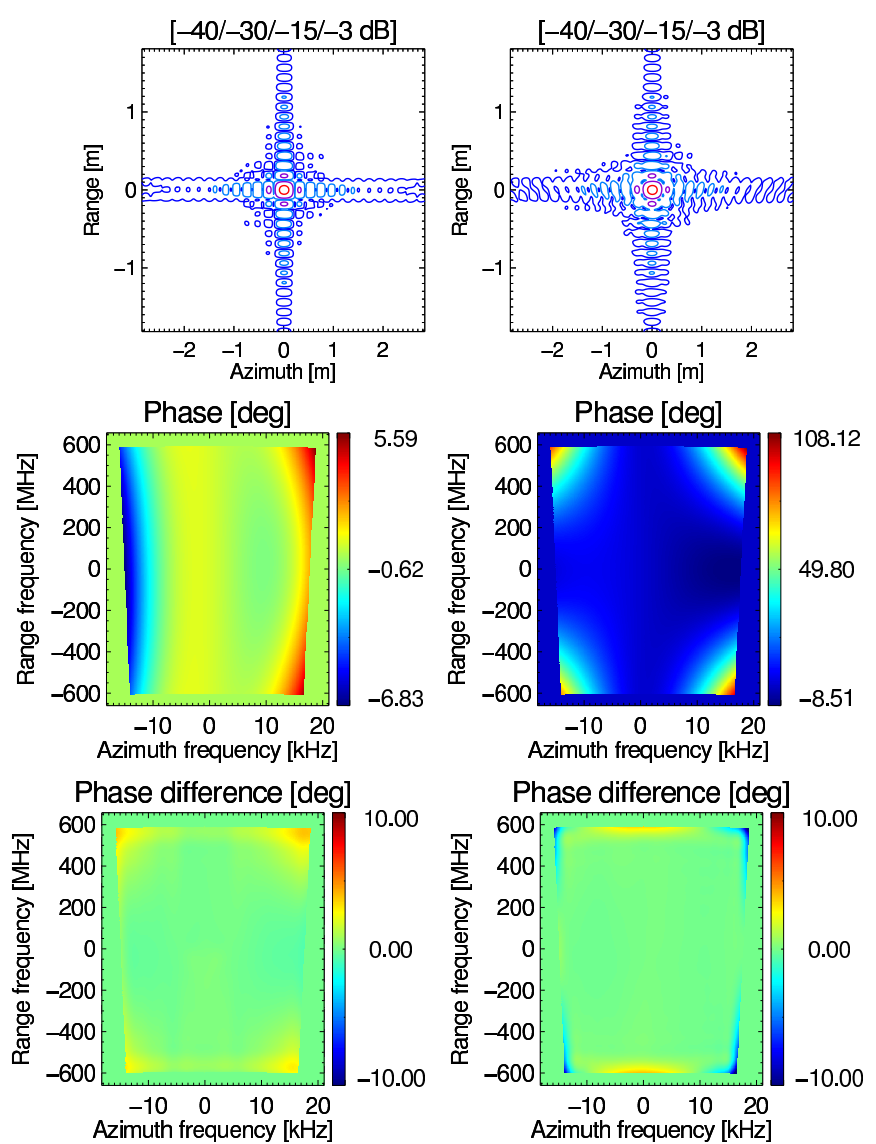

Fig. 3. Comparison between the proposed methodology and the timedomain simulation followed by the true processing for the spotlight example (see Table I). The target is located $2.5 \mathrm{~km}$ away from scene center in the azimuth and ground range dimensions. The plots correspond to (left column) spaceborne EOK [11] and (right column) range-Doppler processors, respectively. (Top row) contour plot of the simulated IRF. (Middle row) Computed phase error in the 2D frequency domain. (Bottom row) Phase difference between the proposed methodology and the true processing.
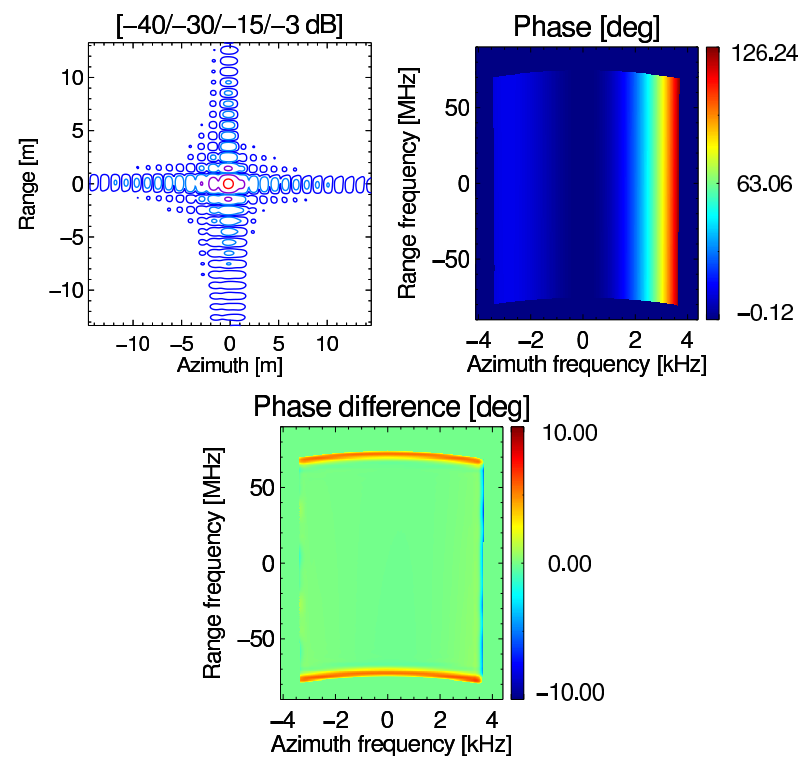

Fig. 4. Comparison between the proposed methodology and the time-domain simulation followed by the true processing for the bistatic example (see Table I). The target is located $125 \mathrm{~m}$ away from scene center in the azimuth dimension. (Top left) Contour plot, (top right) phase error of the kernel in the 2D frequency domain, and (bottom) phase difference between the proposed methodology and the true processing. the mission requirements. Additionally, the concept can be exploited to develop and efficiently test new focusing kernels, hence becoming a valuable complement to the conventional evaluation of a processor via time-domain simulation of point targets. Furthermore, considering that here only the basic idea has been presented, it is straightforward to extend the concept in order to include further aspects like system effects (e.g., replica, antenna pattern), atmospheric effects, the platform motion in the airborne case, etc.

A high resolution spotlight spaceborne geometry and a hybrid bistatic geometry have been used to validate the proposed approach. In both cases the computed responses were practically identical to those obtained with time-domain simulations followed by the true processing.

\section{REFERENCES}

[1] R. Bamler, "A comparison of range-doppler and wavenumber domain SAR focusing algorithms," IEEE Trans. Geosci. Remote Sens., vol. 30, no. 4, pp. 706-713, Jul. 1992.

[2] A. Moreira, J. Mittermayer, and R. Scheiber, "Extended chirp scaling algorithm for air- and spaceborne SAR data processing in stripmap and ScanSAR imaging modes," IEEE Trans. Geosci. Remote Sens., vol. 34, no. 5, pp. 1123-1136, Sep. 1996.

[3] R. Lanari, M. Tesauro, E. Sansosti, and G. Fornaro, "Spotlight SAR data focusing based on a two-step processing approach," IEEE Trans. Geosci. Remote Sens., vol. 39, no. 9, pp. 1993-2004, Sep. 2001.

[4] J. Mittermayer, A. Moreira, and O. Loffeld, "Spotlight SAR data processing using the frequency scaling algorithm," IEEE Trans. Geosci. Remote Sens., vol. 37, no. 5, pp. 2198-2214, Sep. 1999.

[5] P. Prats, R. Scheiber, J. Mittermayer, A. Meta, and A. Moreira, "Processing of sliding spotlight and TOPS SAR data using baseband azimuth scaling," IEEE Trans. Geosci. Remote Sens., vol. 48, no. 2, pp. 770-780, Feb. 2010.

[6] I. G. Cumming and F. H. Wong, Digital Processing of Synthetic Aperture Radar Data. Algorithms and Implementation. Boston, London: Artech House, 2005.

[7] Y. L. Neo, F. Wong, and I. G. Cumming, "A two-dimensional spectrum for bistatic SAR processing using series reversion," IEEE Geosci. Remote Sens. Lett., vol. 4, no. 1, pp. 93-96, Jan. 2007.

[8] D. D'Aria and A. Monti Guarnieri, "High-resolution spaceborne SAR focusing by SVD-Stolt," IEEE Geosci. Remote Sens. Lett., vol. 4, no. 4, pp. 639-643, Oct. 2007.

[9] M. Born and E. Wolf, Principles of Optics. Pergamon, Oxford, $5^{\text {th }}$ Edition, 1975.

[10] C. Cafforio, C. Prati, and F. Rocca, "SAR data focusing using seismic migration techniques," IEEE Trans. Aerosp. Electron. Syst., vol. 27, no. 2, pp. 194-207, Mar. 1991.

[11] P. Prats, R. Scheiber, M. Rodriguez-Cassola, J. Mittermayer, S. Wollstadt, F. D. Zan, B. Bräutigam, M. Schwerdt, A. Reigber, and A. Moreira, "On the processing of very high-resolution spaceborne SAR data," IEEE Trans. Geosci. Remote Sens., 2014, to be published.

[12] G. Fornaro, E. Sansosti, R. Lanari, and M. Tesauro, "Role of processing geometry in SAR raw data focusing," IEEE Trans. Aerosp. Electron. Syst., vol. 38, no. 2, pp. 441-454, Apr. 2002.

[13] G. W. Davidson and I. Cumming, "Signal properties of spaceborne squint-mode SAR," IEEE Trans. Geosci. Remote Sens., vol. 35, no. 3, pp. 611-617, May 1997.

[14] R. Bamler, F. Meyer, and W. Liebhart, "Processing of bistatic SAR data from quasi-stationary configurations," IEEE Trans. Geosci. Remote Sens., vol. 45, no. 11, pp. 3350-3358, Nov. 2007.

[15] A. Reigber, E. Alivizatos, A. Potsis, and A. Moreira, "Extended wavenumber domain SAR focusing with integrated motion compensation," IEE Proc. Radar Sonar Navig., vol. 153, no. 3, pp. 301-310, Jun. 2006.

[16] M. Rodriguez-Cassola, S. V. Baumgartner, G. Krieger, and A. Moreira, "Bistatic TerraSAR-X/F-SAR spaceborne-airborne SAR experiment: description, data processing, and results," IEEE Trans. Geosci. Remote Sens., vol. 48, no. 2, pp. 781-794, 2010. 